\title{
Sonoporation by low-frequency and low-power ultrasound enhances chemotherapeutic efficacy in prostate cancer cells in vitro
}

\author{
YU WANG, WEN-KUN BAI, E. SHEN and BING HU \\ Department of Ultrasound in Medicine, Shanghai Jiao Tong University Affiliated Sixth People's Hospital, \\ Shanghai Institute of Ultrasound in Medicine, Shanghai 200233, P.R. China
}

Received December 23, 2012; Accepted April 17, 2013

DOI: $10.3892 / 01.2013 .1389$

\begin{abstract}
Combination therapy is used to optimize anticancer efficacy and reduce the toxicity and side-effects of drugs upon systemic administration. Ultrasound (US) combined with microbubbles (UM) enhances the intracellular uptake of cytotoxic drugs by tumor cells, particularly drug-resistant cells. In the present study, low-frequency and low-energy US (US irradiation conditions: frequency, $21 \mathrm{kHz}$; power density, $0.113 \mathrm{~W} / \mathrm{cm}^{2}$; exposure time, 2 min at a duty cycle of $70 \%$; and valid treatment time, $84 \mathrm{sec}$ ) were used in combination with microbubbles $(100 \mu \mathrm{l} / \mathrm{ml})$ to deliver mitoxantrone $\mathrm{HCl}$ (MIT) to DU145 cells. The results showed that UM did not change the cell viability in the short- or long-term. However, UM statistically enhanced the therapeutic effects and up to $31.26 \pm 3.34 \%$ of the cells exposed to UM were permeabilized compared with $9.74 \pm 2.55 \%$ of cells in the control, when using calcein (MW, 622.53) as a fluorogenic marker. Notably, UM affected the migration capability of the DU145 cells at $6 \mathrm{~h}$ post-treatment. In conclusion, the ultrasonic parameters used in the present study enhanced the chemotherapeutic effect and reduced the unwanted side-effects of MIT.
\end{abstract}

\section{Introduction}

As an established therapeutic method, ultrasound (US) is used for bone fracture healing, hyperthermia and the ablation of solid tumors (1). Furthermore, in this newly emerging field, US-mediated microbubble destruction, a noninvasive approach, has been shown to possess significant potential to increase the permeability of cell membranes and tissues to various substances. Since US-mediated microbubble destruction is able to reversibly disrupt biological barriers, particularly cell membranes, large quantities of molecules may

Correspondence to: Dr Bing Hu, Department of Ultrasound in Medicine, Shanghai Jiao Tong University Affiliated Sixth People's Hospital, Shanghai Institute of Ultrasound in Medicine, 600 Yishan Road, Shanghai 200233, P.R. China

E-mail: binghu1314@hotmail.com

Key words: low-frequency ultrasound, low-energy ultrasound, microbubble, mitoxantrone delivery then be delivered into tumor cells, particularly drug-resistant cells. The mechanism by which this occurs is considered to be sonoporation, resulting from oscillations of the gas bubbles in the media, which cause cavitation close to the cell surface and subsequent membrane disruption that allows increased drug internalization (2). It has been demonstrated that intracellular uptake is greatly enhanced by diagnostic microbubbles used for US imaging (3-5). At particular ultrasonic frequencies, microbubbles have been shown to greatly enhance transient sonoporation (6). These microbubbles, oscillating in the presence of US, create localized shear stress or 'microstreaming' or they may expand and collapse ('transient cavitation') to create intense local heating and pressure (7). This type of transient cavitation effect is considered to occur more at low frequencies (8). Schlicher et al demonstrated transient pores ( $<28 \mathrm{~nm}$ diameter) in the plasma membrane of cells, following exposure to low frequency US $(24 \mathrm{kHz})(9)$.

Prostate cancer (PCa) is one of the most common types of cancer among the male population of Western countries, second only to skin cancer $(10,11)$. Hormonal therapy allows long-lasting and effective control of cancer-related symptoms at advanced stages. However, in almost all patients with metastatic $\mathrm{PCa}$, the disease progresses when it becomes castration-resistant (CRPC) (12). At that stage, second-line endocrinal therapy and chemotherapy should be administered. In order to maintain sufficient doses of chemotherapeutic drugs in the cancerous tissue, all tissues are exposed to various concentrations of cytotoxic drugs during systemic administration. Combination therapy is used to optimize anticancer efficacy and reduce the toxicity and side-effects of drugs upon systemic administration. US combined with microbubbles (UM) is able to enhance the intracellular uptake of the cytotoxic drugs by the tumor cells, particularly the drug-resistant cells.

Sonoporation, electroporation, microinjection and laser irradiation are all able to enhance the transmembrane delivery of therapeutic molecules. However, sonoporation is considered to be a 'gentle' technique. Non-inertial cavitation is generated by the alternate growth and shrinkage caused by contrast agents (CAs) oscillating, which occurs at low acoustic pressures. Further complex non-linear interactions appear when the ultrasonic pressure reaches a certain threshold, and at that time the microbubble explodes and pores form, which enhances the intracellular uptake or kills the cells. Overall, 
inertial cavitation occurs at relatively high pressure amplitudes and CAs contract and collapse 'violently' (13). Khanna et al, who performed the first notable study, used US waves to make blood cells release hemoglobin (14). The study into US waves by Kinoshita and Hynynen showed that increasing sonoporation typically lessened cellular viability (15). However, Rodamporn et al concluded that improved conditions reduced the loss of viability while maintaining high transfection rates (16). To the best of our knowledge, the present study is the first to state that low-frequency and low-energy US are able to reduce the loss of viability while maintaining high sonoporation in DU145 PCa cells.

\section{Materials and methods}

Cell culture. DU145 cells, a human PCa cell line, were obtained from the Cell Bank of the Chinese Academy of Sciences (Shanghai, China) and used to study the chemotherapy response at the cellular level. The cells were maintained in Dulbecco's modified Eagles medium (DMEM; Gibco, Grand Island, NY, USA) supplemented with $10 \%$ heat-inactivated fetal bovine serum (FBS; Invitrogen, Carlsbad, CA, USA) in $5 \% \mathrm{CO}_{2}$ humidified air at $37^{\circ} \mathrm{C}$. This study was approved by the ethics committee of Shanghai Jiao Tong University Affiliated Sixth People's Hospital (Shanghai, China).

Drugs. Mitoxantrone HCl (MIT; Sigma Aldrich, St. Louis, MO, USA) was dissolved in phosphate-buffered saline (PBS) at a concentration of $10 \mathrm{nM}$ and the solution was stored at $-20^{\circ} \mathrm{C}$ until use. MIT, which was approved by the FDA for hormone refractory PCa (HRPC) as a palliative treatment in 1996, is a cell cycle nonspecific agent that inhibits nucleic acid synthesis, leading to cell death. The dose limiting toxic effects are bone marrow suppression, leukopenia and thrombocytopenia.

US apparatus. FS-450 ultrasonic processing (Shanghai Institute of Ultrasound in Medicine, Shanghai, China) with a SonoVue ${ }^{\mathrm{TM}}$ microbubble echo-contrast agent (Bracco SpA, Milan, Italy) was employed as previously described (17). The US irradiation conditions were as follows: frequency, $21 \mathrm{kHz}$; power density, $0.113 \mathrm{~W} / \mathrm{cm}^{2}$; instrument exposure time, $2 \mathrm{~min}$ at a duty cycle of $70 \%$ (i.e., $7 \mathrm{sec}$ 'on' time and $3 \mathrm{sec}$ 'off' time); and a valid treatment time of $84 \mathrm{sec}$.

Treatment. The cell suspensions were divided into four treatment groups: Group A, non-treated (control); group B, UM treatment; group C, MIT treatment; and group D, combined treatment with MIT and UM (MIT+UM). DU145 cells $\left(1 \times 10^{6}\right.$ in $1 \mathrm{ml}$ of medium) were transferred into $1.5-\mathrm{ml}$ polystyrene test tubes, the diameter of which was the same as the probe being used. MIT (10 nmol) was added to groups C and D.

Analysis of cell proliferation. Immediately following exposure to US, the cells were added to a 96-well plate at a density of 5,000 cells/well and incubated with $5 \% \mathrm{CO}_{2}$ at $37^{\circ} \mathrm{C}$. Subsequent to $24 \mathrm{~h}, 50 \mu \mathrm{l}$ dilution medium containing $10 \mu \mathrm{l}$ MTT solution $(5 \mathrm{mg} / \mathrm{ml})$ was used to replace the medium, and the plate was incubated for another $4 \mathrm{~h}$ until the liquid was removed. The formazan crystals that formed were

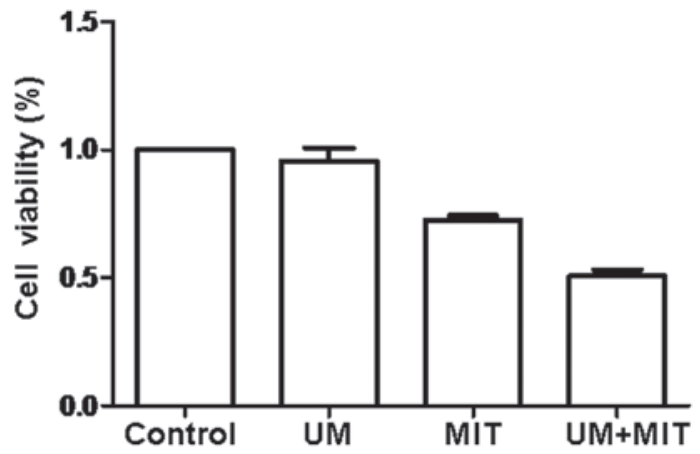

Figure 1. MTT assay showing the cell viability of each group at $24 \mathrm{~h}$ post-treatment $(n=3)$. There was no significant effect on cell viability with UM alone. The viability of cells exposed to MIT+UM was significantly decreased $(50.7 \%)$ compared with those exposed to MIT alone $(72.3 \%$; $\mathrm{P}<0.05)$, indicating that UM enhanced the chemotherapeutic efficacy of MIT in the DU145 cell line $(\mathrm{P}<0.05)$. Bars represent SEM. UM, ultrasound in combination with microbubbles; MIT, mitoxantrone $\mathrm{HCl}$

dissolved with $150 \mu 1$ DMSO. Following agitation for $5 \mathrm{~min}$, the absorbance of each well at a test wavelength of $570 \mathrm{~nm}$ was measured with a microculture plate reader (Bio-Tek, Winooski, VT, USA). The percentage cell viability was calculated as $\mathrm{OD}_{\text {exposed }} / \mathrm{OD}_{\text {control }} \mathrm{x} 100$.

Clonogenic assay. The long-term proliferation rate of the treated cells was measured by plate clonogenic assays. Subsequent to therapy, 200 cells/well were seeded into a 12-well plate, then incubated for two weeks to form colonies prior to fixation in $70 \%$ ethanol, staining with crystal violet and counting.

Cell migration assay. The transwell apparatus (Costar, Cambridge, MA, USA) was used for the cell migration assays. There were $1 \times 10^{4}$ cells in $100 \mu 1$ DMEM without FBS in the the upper polycarbonate membrane insert (pore size, $8 \mu \mathrm{m}$ ), which was precoated with $24 \mathrm{mg} / \mathrm{ml}$ Matrigel (R\&D Systems, Minneapolis, MN, USA), and there was $600 \mathrm{ml}$ DMEM with $10 \% \mathrm{FBS}$ in the lower chamber.

Following incubation for $8 \mathrm{~h}$ at $37^{\circ} \mathrm{C}$ in a $5 \% \mathrm{CO}_{2}$ atmosphere, the upper cells were removed with a cotton swab and the cells adhering to the lower surface were fixed with 95\% alcohol for 15-20 min and stained with crystal violet for $15 \mathrm{~min}$. Finally, the total number of migratory cells was counted with a microscope.

Analysis of DU145 cell permeability using calcein with or without UM. Calcein was used as the permeability tracer to further support our hypothesis that UM increases the absorption of drugs. Calcein $(1 \mu 1,25 \mathrm{mg} / \mathrm{ml}$; Sigma Aldrich) was added to each sample just prior to exposure. Following incubation for $1 \mathrm{~h}$ at $37^{\circ} \mathrm{C}$, the samples were washed three times with PBS, then 10,000 cells/sample were detected by flow cytometry using the CellQuest Pro software program (BD Biosciences, Franklin Lakes, NJ, USA) with an excitation wavelength of $492 \mathrm{~nm}$ and an emission wavelength of $518 \mathrm{~nm}$ (18). The results are expressed as the percentage of positive cells and fluorescence intensity with regard to the whole cell population, including any dead cells. 

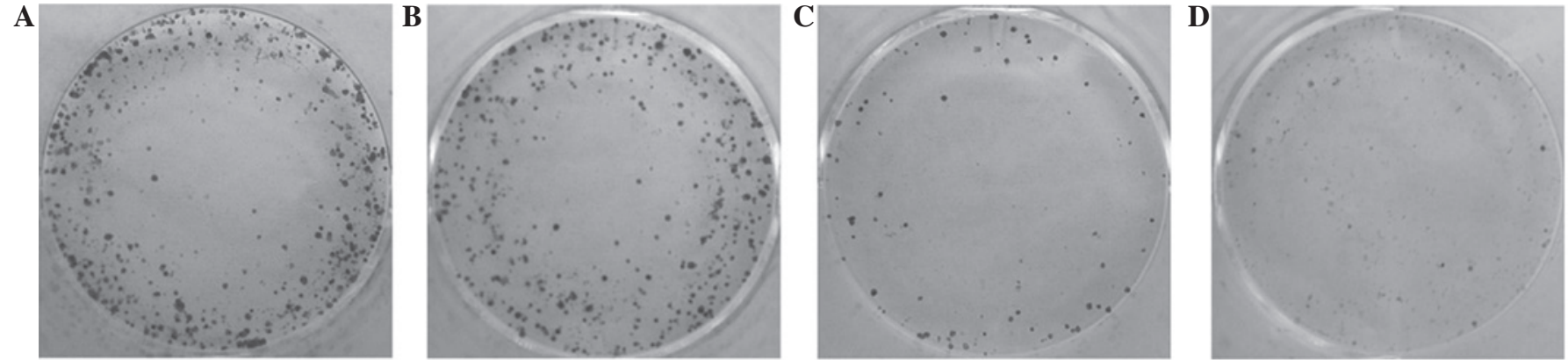

Figure 2. Images of the colony formation of the various groups. (A) Control group; (B) group administered with MIT; (C) group administered with UM; (D) group administered with MIT+UM. The DU145 cells fixed in 70\% ethanol were stained with crystal violet. The clonogenicity of the cells exposed to UM was lower compared with the untreated cells and the numbers of each were $95.7 \pm 7.1$ and $102.0 \pm 6.6$ colonies, respectively (P>0.05). By contrast, the cells exposed to MIT and MIT combined with UM maintained their colony forming ability, with $45.0 \pm 6.0$ and $18.7 \pm 5.5$ colonies, respectively (P<0.05). Crystal violet staining. UM, ultrasound in combination with microbubbles; MIT, mitoxantrone $\mathrm{HCl}$.
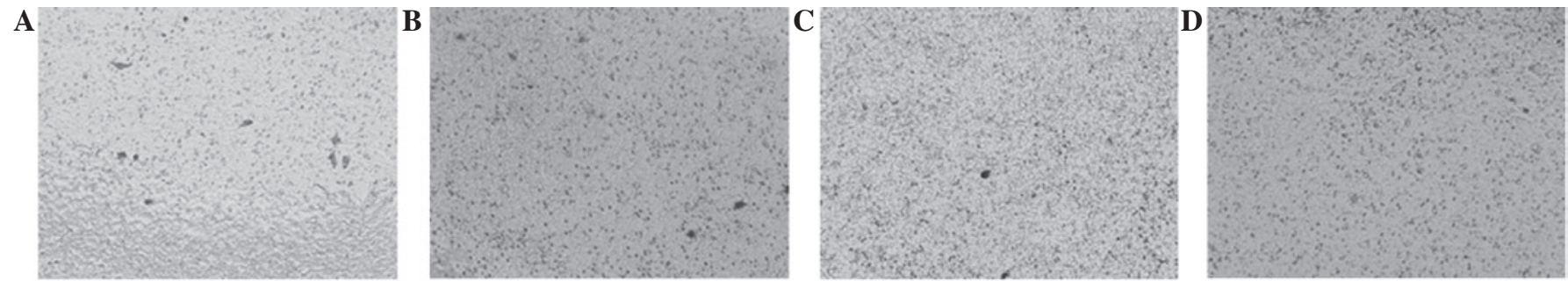

Figure 3. Representative cell migration numbers in human prostate cancer cells. Migration in the (A) control; (B) UM-treated; (C) MIT-treated; and (D) MIT+UM-treated groups. At $6 \mathrm{~h}$ post-treatment, UM, MIT and UM+MIT decreased the migration ability of the DU145 cells (P<0.05). The addition of UM resulted in significant suppression of DU145 migration compared with MIT alone, by $2.7 \pm 2.5$ and 17.7 \pm 7.1 , respectively ( $<<0.05$ ), as compared with the control

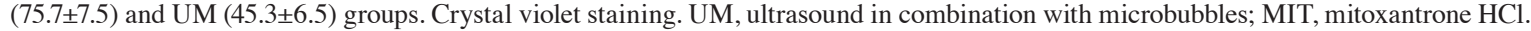
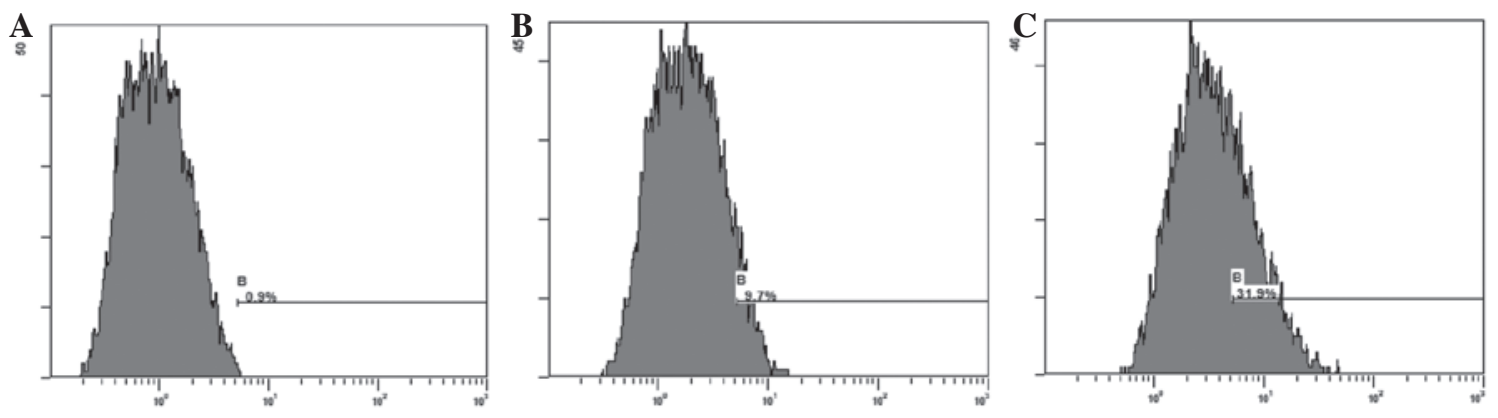

Figure 4. The mean percentage of fluorescence-positive cells in control at $1 \mathrm{~h}$ was $9.74 \pm 2.55 \mathrm{vs.} 31.26 \pm 3.34 \%$ in cells exposed to UM (P<0.05). UM, ultrasound in combination with microbubbles. A, blank control group; B, ultrasound; C, UM.

Statistical analysis. Data are expressed as the mean \pm SEM. The differences among the groups were analyzed using Student's t-test or one-way ANOVA. $\mathrm{P}<0.05$ was considered to indicate a statistically significant difference.

\section{Results}

Increasing the efficacy of chemotherapy (tests of cell proliferation). In order to examine the hypothesis that UM is able to enhance the efficacy of chemotherapy, the cytotoxicity on each group of the cells from the PCa cell line DU145 was evaluated using the MTT assay. Fig. 1 shows the results following incubation for $24 \mathrm{~h}$, which indicated that the MIT+UM group had clear cytotoxicity compared with the other three groups. As shown in Fig. 1, compared with the controls, the cell viability of the UM group did not decrease ( $\mathrm{P}>0.05)$, although the cell viability of the MIT (72.3\%) and MIT+UM (50.7\%) groups was significantly decreased $(\mathrm{P}<0.05)$. Compared with the MIT group, the cell viability of the MIT+UM group was significantly decreased $(\mathrm{P}<0.05)$.

Viability of the reversibly permeabilized cells. The long-term effects of MIT and UM, alone or in combination, on the DU145 cells were evaluated with the colony formation assay. The numbers of cell colonies formed are shown in Fig. 2. The clonogenicity of the cells exposed to UM $(95.7 \pm 7.1)$ was lower compared with the untreated cells $(102.0 \pm 6.6)$ (Fig. 2).

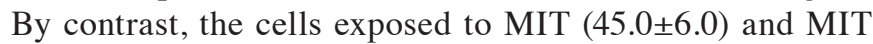
combined with UM $(18.7 \pm 5.5)$ had significantly lower colony forming numbers $(\mathrm{P}<0.05)$. 
Effects on the migration of the DU145 cells. The cell migration ability was assayed using the transwell apparatus in order to investigate the long-term biological effects of each treatment on the DU145 cells. The transmembrane cells of each group are shown in Fig. 3. Compared with the control group, the invasive ability of the DU145 cells in the UM (45.3 \pm 6.5$)$, MIT (17.7 \pm 7.1$)$ and MIT+UM (2.7 \pm 2.5$)$ groups was significantly decreased (all $\mathrm{P}<0.05$; Fig. 3). The results indicated that UM may enhance the ability of MIT in decreasing the colony forming ability of cells.

Analysis of sonoporation efficacy. Fig. 4 shows the percentage of intracellular calcein (a molecular fluorescent probe; MW, 622.53) induced by sonoporation. The cells treated with UM (31.26 $\pm 3.34 \%)$ showed an increased percentage of fluorescence-positive cells compared with the control cells $(9.74 \pm 2.55 \%)$ following incubation for $1 \mathrm{~h}(\mathrm{P}<0.05)$.

\section{Discussion}

Numerous side-effects follow effective MIT therapy. Combination therapy is used to optimize anticancer efficacy and reduce the toxicity and side-effects of drugs upon systemic administration. With its noninvasive and steerable nature, US is a useful tool in combined treatment with anti-cancer agents. Sonoporation has been associated with enhanced drug delivery in chemotherapy in primary studies concerning cutaneous melanoma $(19,20)$ lymphoma $(21)$ and oral cancer $(22,23)$. It appears that there have been few studies focusing on $\mathrm{PCa}$ and combination therapy, and only a small number on the enhancement of permeability in vivo (24).

The present study is the first to investigate this strategy in PCa. The results revealed the successful enhancement of transmembrane MIT transport with the aid of UM, while simultaneously avoiding significantly affecting cell viability. This low frequency and low power-based method did not allow the microbubbles to make contact with the cell membrane immediately and did not exert significant additional stress on the cell membrane to cause death-induced pores. Notably, the study demonstrated for the first time that combining these strategies facilitated the uptake of MIT by DU145 cells in a number of ways. This alternative low-cost device may offer some quality of life improvement for patients, rather than other more expensive techniques, which may be welcomed by individuals in low-level income brackets. Further studies to gain a greater understanding of the mechanisms should be performed prior to this therapeutic method being used in clinical practice, to identify the most appropriate UM parameters that increase the efficacy of all types of therapeutic agents in vivo and are safe for normal tissues. The present study represents a first step towards combination therapy for PCa.

\section{Acknowledgements}

The present study was supported by the major infrastructure projects of Shanghai Science and Technology under grant No. 10JC1412600 and by the National Natural Science Foundation of China (project number, 81271597).

\section{References}

1. Hensel K, Mienkina MP and Schmitz G: Analysis of ultrasound fields in cell culture wells for in vitro ultrasound therapy experiments. Ultrasound Med Biol 37: 2105-2115, 2011.

2. Husseini GA and Pitt WG: The use of ultrasound and micelles in cancer treatment, J Nanosci Nanotechnol 8: 2205-2215, 2008.

3. Casey G, Cashman JP, Morrissey D, et al: Sonoporation mediated immunogene therapy of solid tumors. Ultrasound Med Biol 36: 430-440, 2010.

4. Qin S, Caskey CF and Ferrara KW: Ultrasound contrast microbubbles in imaging and therapy: physical principles and engineering. Phys Med Biol 54: R27-R57, 2009.

5. van Wamel A, Kooiman K, Harteveld M, et al: Vibrating microbubbles poking individual cells: drug transfer into cells via sonoporation. J Control Release 112: 149-155, 2006.

6. Ohl CD, Arora M, lkink R, de Jong N, Versluis M, Delius M and Lohse D: Sonoporation from jetting cavitation bubbles. Biophys J 91: 4285-4295, 2006.

7. Juffermans LJ, van Dijk A, Jongenelen CA, Drukarch B, Reijerkerk A, de Vries HE, Kamp O and Musters RJ: Ultrasound and microbubble-induced intra- and intercellular bioeffects in primary endothelial cells, Ultrasound Med Biol 35: 1917-1927, 2009.

8. Hoskins P, Thrush A, Martin K and Whittingam T (eds): Diagnostic Ultrasound: Physics and Equipment. 2nd Edition. Cambridge University Press, New York, NY, 2010.

9. Schlicher RK, Radhakrishna H, Tolentino TP, Apkarian RP, Zarnitsyn V and Prausnitz MR: Mechanism of intracellular delivery by acoustic cavitation. Ultrasound Med Biol 32: 915-924, 2006.

10. Jemal A, Siegel R, Xu J and Ward E: Cancer statistics, 2010. CA Cancer J Clin 60: 277-300, 2010.

11. Siegel R, Ward E, Brawley O and Jemal A: Cancer statistics, 2011: the impact of eliminating socioeconomic and racial disparities on premature cancer deaths. CA Cancer J Clin 61: 212-236, 2011.

12. Carles J, Castellano D, Climent MÁ, Maroto P, Medina R and Alcaraz A: Castration-resistant metastatic prostate cancer: current status and treatment possibilities. Clin Transl Oncol 14: 169-176, 2012.

13. Mellott AJ, Forrest ML and Detamore MS. Physical non-viral gene delivery methods for tissue engineering. Ann Biomed Eng 41: 446-468, 2013.

14. Khanna S, Amso NN, Paynter SJ and Coakley WT: Contrast agent bubble and erythrocyte behavior in a 1.5-MHz standing ultrasound wave. Ultrasound Med Biol 29: 1463-1470, 2003.

15. Kinoshita M and Hynynen K: Key factors that affect sonoporation efficiency in in vitro settings: the importance of standing wave in sonoporation. Biochem Biophys Res Commun 359: 860-865, 2007.

16. Rodamporn S, Harris NR,Beeby SP,Boltryk RJ and Sanchez-Elsner T: HeLa cell transfection using a novel sonoporation system. IEEE Trans Biomed Eng 58: 927-934, 2011.

17. Bai WK, Wu ZH, Shen E, Zhang JZ and Hu B: The improvement of liposome-mediated transfection of pEGFP DNA into human prostate cancer cells by combining low-frequency and low-energy ultrasound with microbubbles. Oncol Rep 27: 475-480, 2012.

18. Hao Q, Liu Q, Wang X, Wang P, Li T and Tong WY: Membrane damage effect of therapeutic ultrasound on Ehrlich ascitic tumor cells. Cancer Biother Radiopharm 24: 41-48, 2009.

19. Lentacker I, Geers B, Demeester J, De Smedt SC and Sanders NN: Design and evaluation of doxorubicin-containing microbubbles for ultrasound-triggered doxorubicin delivery: cytotoxicity and mechanisms involved. Mol Ther 18: 101-108, 2010.

20. Sonoda S, Tachibana K, Uchino E, et al: Inhibition of melanoma by ultrasound-microbubble-aided drug delivery suggests membrane permeabilization. Cancer Biol Ther 6: 1276-1283, 2007.

21. Yoshida T, Kondo T, Ogawa R, et al: Combination of doxorubicin and low-intensity ultrasound causes a synergistic enhancement in cell killing and an additive enhancement in apoptosis induction in human lymphoma U937 cells. Cancer Chemother Pharmacol 61: $559-567,2008$.

22. Iwanaga $\mathrm{K}$, Tominaga $\mathrm{K}$, Yamamoto $\mathrm{K}$, et al: Local delivery system of cytotoxic agents to tumors by focused sonoporation. Cancer Gene Ther 14: 354-363, 2007.

23. Maeda H, Tominaga K, Iwanaga K, et al: Targeted drug delivery system for oral cancer therapy using sonoporation. J Oral Pathol Med 38: 572-579, 2009.

24. Liu Y, Liu Z, Li T and Ye G: Ultrasonic sonoporation can enhance the prostate permeability. Med Hypotheses 74: 449-451, 2010. 